\title{
BMJ Open Development and validation of the Pressure Injury Prevention Barriers questionnaire in hospital nurses in Spain
}

\author{
Maria Dolores Lopez-Franco, Laura Parra-Anguita, Ines Maria Comino-Sanz, \\ Pedro L Pancorbo-Hidalgo (1)
}

To cite: Lopez-Franco MD, Parra-Anguita L, CominoSanz IM, et al. Development and validation of the Pressure Injury Prevention Barriers questionnaire in hospital nurses in Spain. BMJ Open 2020;10:e041376. doi:10.1136/ bmjopen-2020-041376

- Prepublication history and additional material for this paper is available online. To view these files, please visit the journal online (http://dx.doi.org/10. 1136/bmjopen-2020-041376).

Received 06 June 2020 Revised 01 December 2020 Accepted 09 December 2020

Check for updates

(c) Author(s) (or their employer(s)) 2020. Re-use permitted under CC BY-NC. No commercial re-use. See rights and permissions. Published by BMJ.

Nursing, University of Jaén, Jaen, Andalucía, Spain

Correspondence to Dr Pedro L Pancorbo-Hidalgo; pancorbo@ujaen.es

\section{ABSTRACT}

Objective To develop and validate a new questionnaire to measure the nurses' perceptions of the barriers towards the prevention of pressure injuries (PIs) at hospitals.

Design Validation study with mixed methods.

Setting Four university hospitals in southern Spain. Participants The questionnaire was developed based on a literature review. A panel of 14 wound care experts rated the content validity. A sample of 438 nurses (registered nurses and assistant nurses) participated in the survey. Main outcome measures The psychometric properties of the Pressure Injury Prevention Barriers (PIPB) questionnaire evaluated were: content validity, internal consistency reliability and construct validity.

Results The final version of the questionnaire has 25 items grouped into four factors (management and organisation, motivation and priority, knowledge, and staff and collaboration). The confirmatory factor analysis showed good fit and error indices for the model (Comparative Fit Index $=0.92$, root mean square error of approximation=0.074). Cronbach's alpha was 0.90 (overall), and 0.89 (factor 1), 0.75 (factor 2), 0.72 (factor 3) and 0.45 (factor 4). Construct validity was good, demonstrated by the expected association with the scores on patient safety culture and on considering Pls as an adverse effect of hospital stay, but not with attitude score. Conclusion The PIPB questionnaire is an instrument useful for measuring nurses' perceptions of the barriers to Pls prevention. The initial evidence shows that the questionnaire has good content validity, internal consistency and adequate construct validity. Relevance and comprehensiveness need to be assessed in further studies. It can be used both in research and in the evaluation of clinical settings to implementation of Pls preventive programmes in hospitals.

\section{INTRODUCTION}

Pressure injuries (PIs; formerly known as pressure ulcers or decubitus ulcers) are defined as 'localised damage to the skin and underlying soft tissue usually over a bony prominence or related to medical or other devices. The injury occurs as a result of intense and/or prolonged pressure or pressure in combination with
Strengths and limitations of this study

- A 25-item questionnaire, based on a framework from a literature review and a panel of wound care experts for measuring nurses' perceptions of the barriers to the prevention of pressure injuries at hospitals, was developed and validated.

- This study provides initial evidence of the psychometric properties of the questionnaire, tested through the internal consistency reliability, confirmatory factor analysis and construct validity.

- The questionnaire was validated in 438 registered nurses and assistant nurses from four hospitals.

- The questionnaire was not evaluated by the target population in the initial phase of development, so further studies with a representative sample of the target population are needed to assess the relevance, comprehensiveness and comprehensibility. Neither the temporal stability through a test-retest procedure nor the content validity of the final version was assessed.

shear'. ${ }^{1}$ PIs are considered as a major health problem, with a high prevalence in hospitals in both developed and developing countries. In Europe, the PIs prevalence range from $4.6 \%$ to $27.2 \%^{23}$; in Australia $3 \%$ for inpatient wards and $11.5 \%$ for intensive care units. ${ }^{4} \mathrm{~A}$ recent study reported a prevalence of $3.38 \%$ from hospitals in China. ${ }^{5}$ In countries with weak health systems, the PIs prevalence is higher, such as Indonesia $(10.8 \%),{ }^{6}$ Ethiopia $(14.9 \%)^{7}$ or Brazil $(40 \%$ in an emergency hospital unit). ${ }^{8}$ In other settings, such as palliative care, high prevalence figures have been reported: $18.8 \%$ for cancer patients at home in Brazil, ${ }^{9} 16.7 \%$ in Spain $^{3}$ or $13.1 \%$ in home palliative care in Italy. ${ }^{10}$

PIs have a major impact on the quality of life of people who suffer them, causing pain, physical and psychological problems. ${ }^{11}$ The economic impact is also huge. The treatment of hospital-acquired PIs is as high as 
$\$ 26.8$ billion, according to a recent report of US national data. ${ }^{12}$ There is an international consensus on the consideration of PIs as an avoidable adverse event in healthcare (excluding the Kennedy terminal ulcers or other skin changes at life's end, that were considered as unavoidable injuries) ${ }^{13}$; the National Quality Forum has included PIs in a list of reportable serious events categorised as an event that should never occur. ${ }^{14}$

A number of factors have been associated with the development of PIs, some as aetiologic factors (friction, shear, pressure, moisture $)^{15}$ and others as risk factors (age, diabetes, malnutrition, poor circulation, blood disorders) ${ }^{16}$ There are several models or frameworks that establish the relationships between these factors and their effect on the development of PIs. ${ }^{17-20}$

The research on aetiologic and associated factors has been used to inform the recommendations for PIs prevention contained in the guidelines. ${ }^{21}$ Nurses play a major role in implementing these preventive measures, so there is a need to determine nurses' knowledge and attitude towards prevention. ${ }^{22}{ }^{23}$ Nurses should acquire a set of skills and competencies for PIs prevention within the curriculum, including an up-to-date knowledge and a positive attitude. ${ }^{24}$ Recent research proposes that the prevention of PIs at hospitals is a complex phenomenon that involves, not only nurses' characteristics, but also environmental factors (such as nursing workload, staffing and resources). ${ }^{25}$

Many of these external factors may act or be perceived as barriers for effective PIs prevention, as described in different settings and countries. ${ }^{26-29} \mathrm{~A}$ recent literature review found up to four instruments aimed at identifying barriers towards PIs prevention ${ }^{30}$; but most of them are focused on measuring knowledge or attitudes and only list potential barriers as open-ended questions, so none of them have enough evidence of reliability and validity. This is the case with the Moore and Price's instrument that is focused on measuring attitudes and which included three open questions about barriers. ${ }^{27}$

The purpose of this study was to develop and validate a new tool to measure the nurses' perception of the barriers that hinder the prevention of PIs at hospitals, based on updated guidelines and recommendations. ${ }^{21}{ }^{22}$ This instrument was developed as part of a research project named 'Pressure injuries as adverse events: patient safety issues, knowledge, attitudes and perceived barriers by nurses in Spain' (SECOACBA project) ${ }^{31}$ that was aimed to explore the relationships among these factors at hospitals in Spain.

\section{METHODS}

\section{Design}

The study was carried out in three stages:

1. Construction of the questionnaire.

2. Content validation by an expert panel.

3. Psychometric testing of the new questionnaire developed in a sample of nurses.

\section{Construction of the questionnaire and selection of items}

The process started with a literature review aimed at identifying relevant articles about the construct 'barriers or difficulties to PIs prevention'. Thirteen bibliographic databases were searched until 31 November 2016 for articles with any design that focused on barriers to the prevention of PIs, published in English, Spanish, Italian or Portuguese.

Eighteen articles met the inclusion criteria and were selected for the review. From these 18 articles, all the individual factors or situations that were mentioned as a possible barrier for PIs prevention were extracted. Most of the potential barriers were related to the hospital setting. The Beitz quality wound care model ${ }^{32}$ and the Reason adverse event mode ${ }^{33}$ were used as a framework to group these barriers into similar themes. The Beitz's model for wound care quality is based on the general systems theory and proposed three level for barriers: individual (inadequate patient knowledge, deleterious lifestyle habits, poor personal health accountability), group (poor clinicians knowledge, lack of meaningful organisation, economic constraints, poor quality improvement process, lack of research-based guideline, lack of clinicians' research mind frame) and societal (national nursing shortage, lack of populations perspective, lack of national wound benchmarks). ${ }^{32}$ Reason proposed a system approach for adverse effects in healthcare (named the Swiss cheese model of system accidents) with two set of factors: active failures and latent conditions (such as time pressure understaffing, inadequate equipment or inexperience). ${ }^{33}$ Most of the barriers to PIs prevention would be latent conditions. A list of 28 items identifying potential barriers was prepared and grouped into seven themes: management and leadership (2 items); resources availability (5 items); training and education ( 5 items); beliefs of the nursing professionals (2 items); register and recording (2 items); care delivery (10 items) and patient participation (2 items). This was the first version of the Pressure Injury Prevention Barriers (PIPB) questionnaire, with 28 items, that was used for content validation.

\section{Content validation by an expert panel}

A panel of 14 experts (9 men and 5 women) in PIs and wound care was asked for the content validation. The experts were registered nurses (RNs) members of the Spanish National Advisory Group on Pressure Ulcers and Chronic Wounds, with more than 10 years of experience in wound care from different cities in Spain and from different settings: hospital (9), primary care (3) and university (2).

Each expert evaluated the 28 items according to relevance and clarity using a 5-point scale, from 1 (not relevant-not clear) to 5 (very relevant-very clear). They were asked to propose new items when it was necessary. Aiken's V Index ${ }^{34}$ was used to quantify the consensus among the experts; considering the value of 0.50 as the threshold. 


\section{Psychometric testing}

To evaluate the psychometric properties (validity and reliability) of the new questionnaire, a cross-sectional study was conducted on four public hospitals (two acute, one long-term care and one mother and child hospital) in Jaén (southern Spain) from March to April 2017. The PIPB questionnaire was administered to RNs and assistant nurses (ANs) with more than 6 months of clinical experience and working in units or services providing direct care to patients.

A minimum sample size of 150 was estimated 'a priori' according to the methodological recommendations for the validation of questionnaires (five people per item) ${ }^{35}$; but all the RNs and ANs working in 29 units of the hospitals were invited to participate in order to maximise the sample size and reduce sampling bias.

\section{Data collection}

Data were collected through a paper form that was distributed, with an invitation letter, to all the RNs and ANs working in the 29 hospital units. The variables measured were: demographic, training on PIs prevention, patient safety culture, PIs as an adverse effect, perceived barriers towards PIs prevention and attitude towards PIs prevention. The form for collecting data included: a brief questionnaire on demographic and education background; two questions about the perception of patient safety culture and PIs as an adverse effect of hospital stay; the 30-item version of the PIPB questionnaire (as the main instrument). Additionally, a Spanish version of the Attitude towards Pressure ulcer Prevention (APuP) questionnaire ${ }^{3637}$ was used to rate attitude.

\section{Instruments}

The PIPB questionnaire (version 2) has 30-items about different factors and situations that could be perceived as barriers to PIs prevention. The nursing professionals were asked to rate each item on how often they perceive this barrier to be present in their regular practice. Response options were: never, sometimes, frequently and always.

The APuP questionnaire-the Spanish version developed and validated for this research ${ }^{37}$-has 12 items grouped in five factors; has a good content validity, moderate internal consistency (alpha $=0.70$ ) and good fit indices of the factor structure by confirmatory factor analysis (CFA). It has a 4-points Likert scale from complete disagreement to complete agreement and is aimed to measure the nurses' attitudes towards the prevention.

Patient safety culture in the unit. Direct question to rate how they consider that patient safety culture is applied in their unit (from 0 -minimum to 10-maximum).

PIs as an adverse effect of hospital stay. Direct question to rate how they consider that the development of PIs is an adverse effect related with healthcare (from $0-$ minimum to 10 -maximum).

\section{Data analysis}

Data were analysed using SPSS V.21 (IBM) and MPlus 7 (Muthén \& Muthén, Los Angeles, California, USA). ${ }^{38}$ Participants' characteristics were described by frequencies and percentages or mean and SD. Cases with more than three items in the blank (missing data) in the PIPB questionnaire were excluded for the analysis.

First, descriptive items statistics were estimated (missing values, mean, SD and percentages for each response option). The floor and ceiling effects (more than 15\% of responses in the lowest or highest option ${ }^{39}$ ) were analysed. The adjustment of the total score to a normal distribution was tested using a $\mathrm{Q}-\mathrm{Q}$ graphics.

To elaborate a structure for the questionnaire, the dataset was randomly split into two halves $(n=221$ and $\mathrm{n}=217$ ). The first half was used to explore dimensionality using exploratory factor analysis (EFA) with maximum likelihood extraction. The number of factors to extract was decided by examining the screeplot ${ }^{40}$ and by a parallel analysis with the Horn's criteria ${ }^{41}$ using the MonteCarlo PCA software. ${ }^{42}$ Several rotation methods (varimax, quartimax and equamax) were examined to seek an interpretable structure guided by conceptual meaning of the factors. ${ }^{40}$

Then, the second half of data was used to test the model by CFA using structural equations analysis with MPlus7. The weighted least squares with mean and variance adjustment method was used to estimate the parameters because the items were ordinal. Several fit indices were calculated, relative $\chi^{2}$ ( $\chi^{2}$ divided by the $\mathrm{df}$ ), Comparative Fit Index (CFI), Tucker Lewis Index (TLI), root mean square error of approximation (RMSEA) and weighted root mean square residual (WRMR). Values of good fit of the model are: relative $\chi^{2}<3$ ( $>5$ means poor fit), CFI $>0.90$, TLI $>0.90$, RMSEA $<0.08$ and WRMR $<1 .{ }^{43}$ The values of Modification Index (MI) were revised and the changes included in the model, when they were coherent with the theoretical model.

Internal consistency was calculated by item-total correlation and Cronbach's alpha. ${ }^{44}$ Construct validity was explored by the known-groups test; comparing one group with expected high barriers score versus one group with expected low barriers score by a t-test for independent groups.

\section{Patient and public involvement}

Patients and the public were not involved in the design or conduct of the study.

\section{Ethics}

Data obtained were anonymous according to the Spanish Law of Personal Data Protection. The study was approved by the Director of the participants' hospitals. On the first page of the form, participants were fully informed about the purpose and procedures of the study, and it was stated that the completion and submission of the questionnaires implies an agreement to participate. For the collection of the questionnaires and to guarantee anonymity and 
confidentiality, each unit was provided with a box to collect the sealed envelopes with the completed questionnaires.

\section{RESULTS}

\section{Content validation}

The 28 items about potential barriers to PIs prevention drafted, based on the literature review, formed the version 1 of the questionnaire. All the items achieved enough agreement on relevance and clarity by the experts (table 1). The experts proposed two new items: 'Variability in implementation and poor adherence of care plans' and 'Lack of cooperation from family caregivers at the hospital', that were included in the questionnaire. The PIPB questionnaire with 30 items (version 2) was used to conduct the psychometric analysis in a sample of nursing professionals (table 1 ).

\section{Participant characteristics}

A total of 862 RNs and ANs were invited to participate and received the questionnaires; and 438 returned them filled (response rate: $50.8 \%$ ). The reasons for not participating in the study were not requested nor recorded due to the anonymity of the survey. Table 2 shows the main characteristics of the participants.

\section{Item analysis}

The descriptive statistics of the 30 items are shown in table 3. Four items $(13.3 \%)$ have floor effect and two items $(6.6 \%)$ have ceiling effect; but only the item 1 (shortage of staff to provide preventive care) shows a lot of ceiling effect, because one-third of the responses go to the highest option. The SD shows that there is a substantial amount of variability in responses.

\section{Validity}

\section{Dimensionality}

To examine the dimensionality of the questionnaire an EFA was performed on the first half of data. The data set was adequate for EFA (Kaiser-Meyer-Olkin $=0.861$; Bartlett test $\mathrm{p}<0.0001)$. Examining the scree plot and by the Horn's parallel analysis, the number of factors to extract was fixed in four. The EFA with maximum likelihood extraction with four factors was run and several rotation methods explored. Equamax rotation produced an interpretable model with four factors labelled 1-management and organisation (12 items); 2-motivation and priority (6 items); 3-knowledge (4 items) and 4-staff and collaboration (4 items). Four items showed weak item-total correlation and/or factor loading lower than 0.4 in all factors, so they were excluded for the model (see online supplemental additional file 1). These items were: 10 (Lack of clear management guidelines and institutional objectives in pressure injuries prevention); 11 (Difficulty in implementing preventive measures due to the instability of patients' conditions); 14 (Cost of preventive products and devices) and 19 (Difficulty in accessing scientific literature and bibliographic resources).
The 26-item four-factor model was tested by CFA with the second half of data. The first model showed good fit and error indices (table 4) but the item 1 had high cross loading with all the factors as indicated by values of MI. Because this item had also a high ceiling effect, it was removed. A second model with 25 items was tested and showed a slightly better fit that could be improved with a correlation between items 5 and 7, according the MI. The final model has an acceptable fit (table 4). The path diagram of the factor structure proposed for the PIPB is presented in figure 1.

\section{Construct validity}

Construct validity was explored by known-groups test. The groups were formed based on scores in patient safety culture, PI as an adverse effect of hospital stay and attitude towards PIs prevention. The characteristics of the subgroups were roughly similar, with only some differences in the professional category (see online supplemental additional file 2). The hypotheses tested were:

- A high score in the PIPB questionnaire in nursing professionals who scored low on patient safety culture. Professionals who have a poor perception of the importance of patient safety should consider more barriers to prevention.

- A high score in the PIPB questionnaire in nursing professionals who scored high in PI as an adverse effect. Professionals who considered the PI as an adverse effect of hospital stay should also consider more barriers for prevention.

- A high score in the PIPB questionnaire in nursing professionals who scored low in attitude towards PIs prevention. Professionals who have a less positive attitude towards prevention should consider more barriers.

The test for mean differences (t-test for independent groups) supports two of these hypotheses (table 5); providing some evidence that the PIPB questionnaire is able to differentiate between professionals who perceive more or less barriers for PIs prevention.

\section{Reliability}

The internal consistency of the final version of the PIPB questionnaire (25 items) was Cronbach's alpha $=0.90$ (95\% CI $=0.88$ to 0.92$)$ (overall) and alpha $0.89(95 \%$ $\mathrm{CI}=0.87$ to 0.91$) ; 0.75$ (95\% CI $=0.71$ to 0.79$) ; 0.72(95 \%$ $\mathrm{CI}=0.68$ to 0.77$)$ and $0.45(95 \% \mathrm{CI}=0.35$ to 0.53$)$ for the factors 1, 2, 3 and 4, respectively. No individual item was found to increase the alpha if deleted.

\section{The PIPB questionnaire}

The final version of the PIPB questionnaire has 25 items grouped into four factors. Each item can be rated on a 4-point scale according to how often respondents perceive that the barrier will occur in their usual practice: never (scored 0 points), sometimes (1 point), frequently (2 points) and always ( 3 points). The total score of the PIPB questionnaire was calculated by adding the points for the 
$\widehat{\overbrace{}}$

$\stackrel{\varrho}{\stackrel{0}{\circ}}$

ำ

$\frac{0}{\frac{\partial}{m}}$

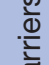

范

을

ญे

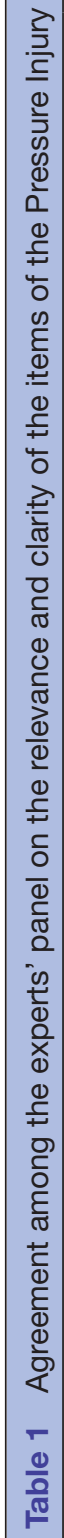

তิ

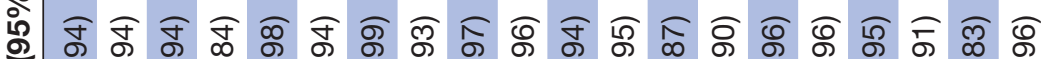

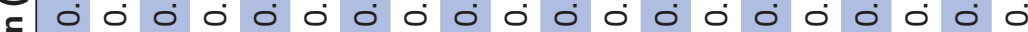

$\stackrel{\varpi}{\S}$

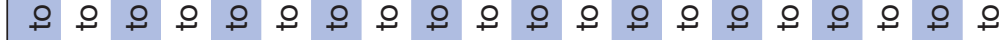

E

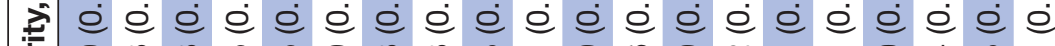

元

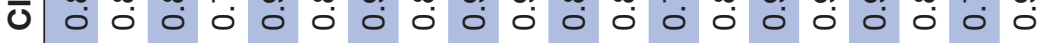

তิ

ㄴํㅇ

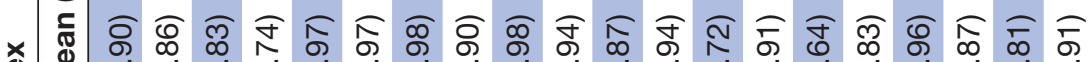

ब

5

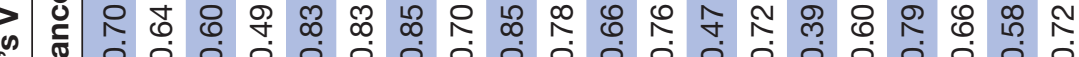

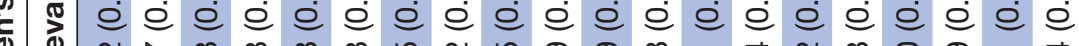
范

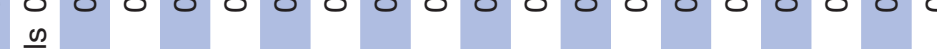

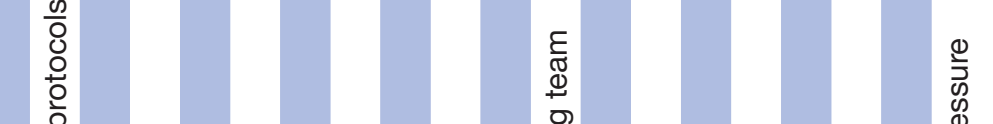

을

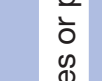

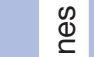

$\frac{.}{\frac{0}{0}}$

$\frac{\frac{0}{0}}{\frac{0}{5}}$

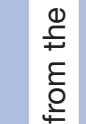

인

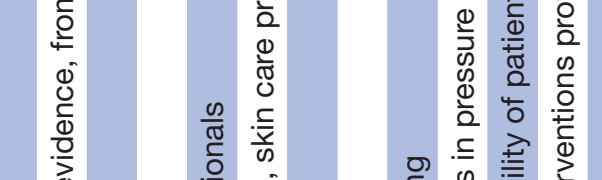

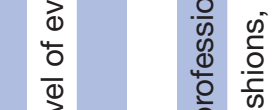

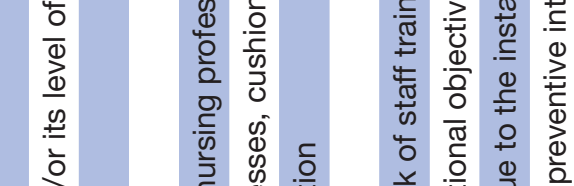

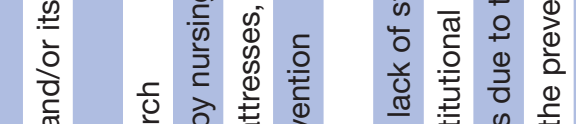

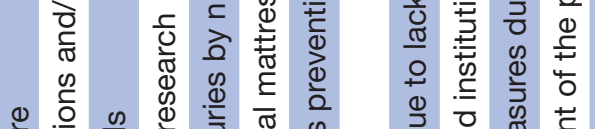

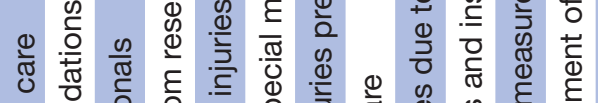

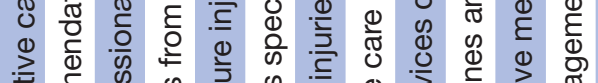

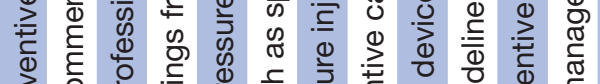

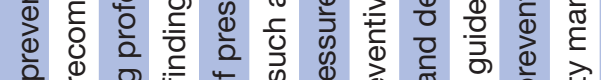

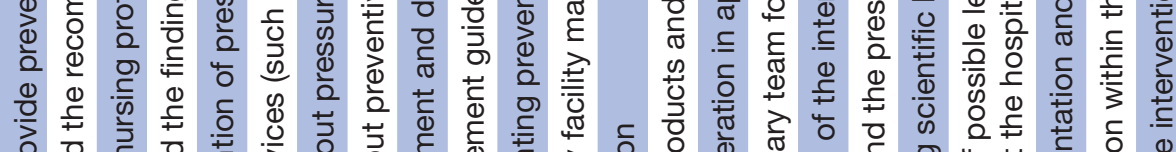

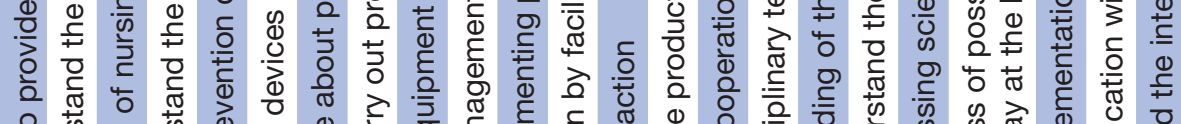

垔

妾

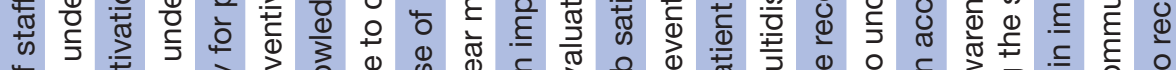

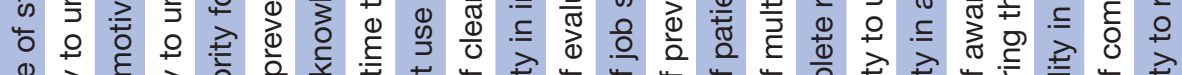
응

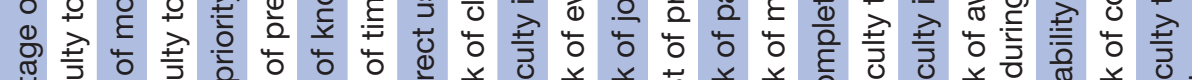

元 至

क

$\stackrel{\mathrm{g}}{=}$

के ले

\& 文 응 응 
Table 2 Sociodemographic characteristics of the sample of nursing professionals $(n=438)$

\begin{tabular}{|c|c|}
\hline Variable & Frequency* (\%) \\
\hline \multicolumn{2}{|l|}{ Gender } \\
\hline Female & $354(80.8)$ \\
\hline Male & $52(11.9)$ \\
\hline \multicolumn{2}{|l|}{ Age (years) } \\
\hline 20-30 & $7(1.6)$ \\
\hline $31-40$ & $57(13.0)$ \\
\hline $41-50$ & $162(37.0)$ \\
\hline $51-60$ & $192(43.8)$ \\
\hline $61-69$ & $16(3.7)$ \\
\hline \multicolumn{2}{|l|}{ Professional category } \\
\hline Registered nurse & $266(60.7)$ \\
\hline Assistant nurse & $161(36.8)$ \\
\hline \multicolumn{2}{|l|}{ Academic degree } \\
\hline Technical training (two years) & $150(34.2)$ \\
\hline Nursing diploma (three years) & $228(52.1)$ \\
\hline Nursing degree (four years) & $27(6.2)$ \\
\hline Bachelor (four years) & $7(1.6)$ \\
\hline Postgraduate: master & $15(3.4)$ \\
\hline Doctorate & $2(0.5)$ \\
\hline \multicolumn{2}{|l|}{ Work experience (years) } \\
\hline$<10$ & $30(6.8)$ \\
\hline $11-20$ & $124(28.3)$ \\
\hline $21-30$ & $176(40.2)$ \\
\hline$>31$ & $104(23.7)$ \\
\hline \multicolumn{2}{|l|}{ Specific training in PI prevention } \\
\hline None & $67(15.3)$ \\
\hline Basic† & $93(21.2)$ \\
\hline Multipleł & $278(63.5)$ \\
\hline
\end{tabular}

*Percentages up to $100 \%$ correspond to missing data. †Acquired in undergraduate.

$\ddagger$ Basic plus conference attendance and/or postgraduate. $\mathrm{PI}$, pressure injury.

25 items and had a range from 0 to 75 points; a higher score means more barriers perceived. Additionally, it is possible to quantify the number of barriers perceived, counting the items with the responses frequently or always (range: 0-25). The PIPB questionnaire and the instructions for scoring can be obtained in the online supplemental additional file 3 .

\section{DISCUSSION}

This study has developed and validated a questionnaire to measure the nurses' perception of the barriers towards the prevention of PIs. This instrument is structured as a reflective model in which the items reflect the latent variable (perception of barriers towards PIs prevention), which is supported by high inter-item correlations and factorial structure. The instrument was named the PIPB questionnaire and has 25 items grouped in four factors: management and organisation, motivation and priority, knowledge, and staff and collaboration. Taking into account the two models used as a framework, these four factors may be considered as latent conditions in the Reason's model. ${ }^{33}$ They are factors that can contribute to the development or occurrence of the problem (a PI) when an active failure happens. In the Beitz model of quality wound care, the barriers in factors 2 (motivation and priority) and 3 (knowledge) fit into the 'group' level related to clinicians, and factors 1 (management and organisation) and 4 (staff and collaboration) fit into the level 'societal' related to the organisation and health system.

Each item can be rated on a 4-points scale, according to how often it is perceived. The PIPB questionnaire has good content validity, internal reliability consistency and construct validity, and is, therefore, an adequate tool to determine the nurses' perception of the barriers towards the prevention of PIs. This questionnaire should not be used as a diagnostic tool to identify the actual barriers present at hospital units, as that requires a different approach.

This new questionnaire is based on a comprehensive literature review and has been evaluated by a panel of experts in wound care to support the relevance and clarity of each item. This expert evaluation is missing in most of the tools for barriers identification described in the literature, ${ }^{30}$ so it is an important feature of this questionnaire. The literature has provided a framework, based on the Beitz ${ }^{32}$ and Reason ${ }^{33}$ works, for the construct. The 'barriers for the prevention of PIs,' we defined as 'A set of elements from the health system (organisation, structure and management) and from individuals (healthcare providers, patients and families) that may prevent the provision of the standard of care in the prevention of PIs.'

Although five items from the initial pool were removed for the final version of the questionnaire, its global comprehensiveness is good enough. These items refer to issues related with management, staff and knowledge that are well covered in the questionnaire. Only one item removed refers to the individual level barriers (patient conditions) that is a level not covered in this instrument. This level could be explored in further studies.

The high internal consistency of the PIPB questionnaire means that all of the items contribute to the global construct measured; the Cronbach's alpha is higher than the questionnaire used by Garza et al (alpha $=0.72)^{45}$ and similar to other instruments, such as Drake et al (alpha $=0.81){ }^{46}$ or Mirshekari et al (alpha $\left.=0.89\right) .{ }^{47}$ Other instruments, such as Moore and Price's questionnaire on attitudes, behaviours and perceived barriers, do not provide a value of internal consistency for the barriers. ${ }^{27}$ When considering the factor structure, three of them have an acceptable or good internal consistency; but the factor 4 'Staff and collaboration' has a poor internal consistency 
Table 3 Item descriptive statistic $(n=438)$

\begin{tabular}{|c|c|c|c|c|c|c|c|}
\hline \multirow[t]{2}{*}{ Item } & \multirow[t]{2}{*}{ Missing (n) } & \multirow[t]{2}{*}{ Mean } & \multirow[t]{2}{*}{ SD } & \multicolumn{4}{|c|}{ Responses values $(\%)^{*}$} \\
\hline & & & & $\begin{array}{l}\text { Never } \\
(0)\end{array}$ & Sometimes (1) & Frequently (2) & Always (3) \\
\hline 1 & 7 & 2.17 & 0.74 & 3.9 & 10.0 & 52.7 & 33.4 \\
\hline 2 & 15 & 1.37 & 0.73 & 9.0 & 50.1 & 35.0 & 5.9 \\
\hline 3 & 8 & 1.31 & 0.78 & 15.8 & 42.1 & 37.7 & 4.4 \\
\hline 4 & 23 & 1.44 & 0.69 & 9.4 & 42.7 & 45.1 & 2.9 \\
\hline 5 & 16 & 1.08 & 0.78 & 23.5 & 47.2 & 25.6 & 3.8 \\
\hline 6 & 17 & 1.64 & 0.87 & 9.5 & 33.7 & 40.4 & 16.4 \\
\hline 7 & 13 & 1.11 & 0.75 & 20.2 & 50.6 & 26.1 & 3.1 \\
\hline 8 & 7 & 1.71 & 0.82 & 8.8 & 26.2 & 50.8 & 14.2 \\
\hline 9 & 12 & 1.35 & 0.77 & 12.7 & 45.1 & 37.1 & 5.2 \\
\hline 10 & 12 & 1.57 & 0.75 & 8.0 & 35.7 & 48.4 & 8.0 \\
\hline 11 & 11 & 1.51 & 0.73 & 8.9 & 37.2 & 48.2 & 5.6 \\
\hline 12 & 14 & 1.46 & 0.75 & 10.1 & 41.0 & 42.7 & 6.1 \\
\hline 13 & 13 & 1.33 & 0.80 & 13.6 & 46.8 & 32.0 & 7.5 \\
\hline 14 & 19 & 1.59 & 0.72 & 7.6 & 33.4 & 52.3 & 6.7 \\
\hline 15 & 12 & 1.33 & 0.67 & 9.2 & 51.9 & 36.2 & 2.8 \\
\hline 16 & 9 & 1.63 & 0.78 & 8.2 & 31.9 & 49.0 & 11.0 \\
\hline 17 & 21 & 1.62 & 0.76 & 7.4 & 33.8 & 48.7 & 10.1 \\
\hline 18 & 11 & 1.22 & 0.70 & 11.9 & 57.6 & 26.7 & 3.7 \\
\hline 19 & 16 & 1.30 & 0.73 & 12.1 & 49.8 & 33.9 & 4.3 \\
\hline 20 & 16 & 1.06 & 0.78 & 19.2 & 58.5 & 18.7 & 3.6 \\
\hline 21 & 13 & 1.41 & 0.81 & 13.6 & 38.8 & 40.5 & 7.1 \\
\hline 22 & 18 & 1.47 & 0.79 & 13.1 & 33.8 & 46.9 & 6.2 \\
\hline 23 & 18 & 1.34 & 0.75 & 11.2 & 50.2 & 31.7 & 6.9 \\
\hline 24 & 16 & 1.34 & 0.77 & 14.5 & 42.7 & 37.9 & 5.0 \\
\hline 25 & 11 & 1.53 & 0.79 & 9.1 & 38.9 & 41.7 & 10.3 \\
\hline 26 & 20 & 1.71 & 0.76 & 7.4 & 27.0 & 53.8 & 11.7 \\
\hline 27 & 13 & 1.72 & 0.76 & 6.4 & 28.5 & 52.2 & 12.9 \\
\hline 28 & 19 & 1.59 & 0.76 & 7.6 & 37.0 & 45.6 & 9.8 \\
\hline 29 & 9 & 1.30 & 0.75 & 13.1 & 49.0 & 32.4 & 5.6 \\
\hline 30 & 6 & 1.59 & 0.83 & 10.9 & 31.5 & 45.8 & 11.8 \\
\hline
\end{tabular}

*Percentage of valid responses for each item.

which may be due to the small number of items and/or to low correlation of some of the items. More studies with larger samples are needed to test this four-factor structure by CFA.
A key strength of this study is that the PIPB questionnaire has been validated in a large sample of RNs and ANs (more than 400) with a robust process of testing its psychometric properties. Although the sample that

Table 4 Confirmatory factor analysis for the Pressure Injury Prevention Barriers questionnaire

$\begin{array}{llllll} & \text { Relative } \chi^{2} & \text { CFI } & \text { TLI } & \text { RMSEA (95\% CI) } & \text { WRMR } \\ \text { Model } 1 \text { (26 items) } & 2.02 & 0.90 & 0.89 & 0.075(0.067 \text { to } 0.084) & 1.24 \\ \text { Model } 2 \text { (25 items) } & 2.05 & 0.91 & 0.90 & 0.076(0.067 \text { to } 0.086) & 1.20 \\ \text { Model } 2 \text { modified }(25 \text { items) } & 1.98 & 0.92 & 0.91 & 0.074(0.064 \text { to } 0.083) & 1.17\end{array}$

CFI, Comparative Fit Index; RMSEA, root mean square error of approximation; TLI, Tucker Lewis Index; WRMR, weighted root mean square residual. 


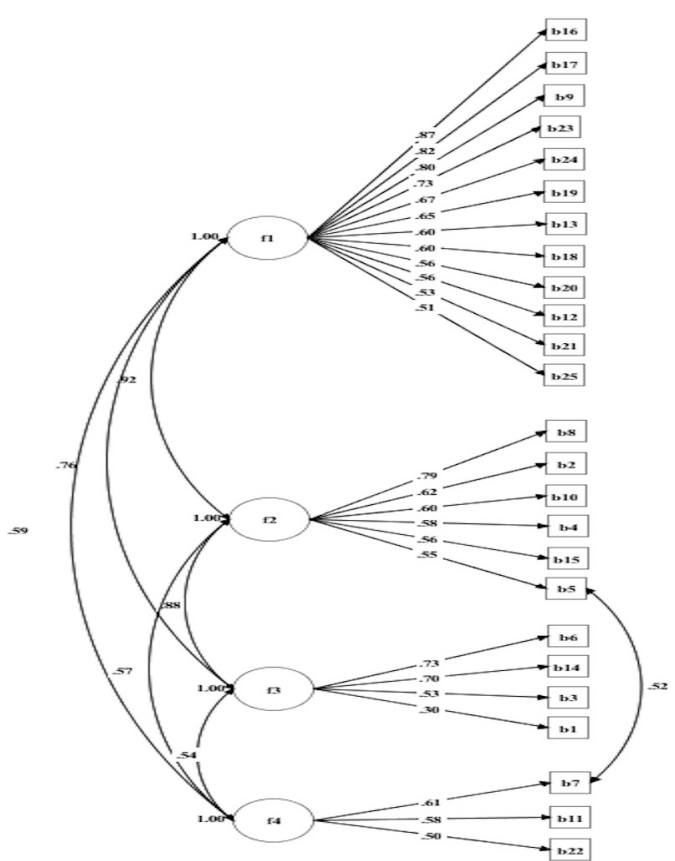

Figure 1 Path diagram of the factor structure of the Pressure Injury Prevention Barriers questionnaire.

participated was not at random, it may be considered representative of the population in terms of age, training and experience, so it is adequate for a validation study. None of the few previously published instruments to measure the barriers to PIs prevention had a formal evaluation of the reliability and construct validity which limits their usefulness in research. ${ }^{26-29} 48$

The four-factor structure of the new questionnaire allows estimating the numbers of perceived barriers related to several aspects of healthcare, such as management, motivation, staffing or collaboration. We consider that it is important to include communication and multidisciplinary teamwork among the potential barriers in

\begin{tabular}{|c|c|c|}
\hline Variable & PIPB score, mean (SD) & $P$ value \\
\hline \multicolumn{3}{|c|}{ Patient safety culture* } \\
\hline Low & $40.24(9.68)$ & \multirow[t]{2}{*}{$<0.0001$} \\
\hline High & $25.96(10.05)$ & \\
\hline \multicolumn{3}{|c|}{$\mathrm{PI}$ as an adverse effect $\dagger$} \\
\hline Low & $27.80(11.04)$ & \multirow[t]{2}{*}{0.002} \\
\hline High & $35.95(12.36)$ & \\
\hline \multicolumn{3}{|c|}{ Attitude towards Pls preventionł } \\
\hline Low & $37.54(9.09)$ & \multirow[t]{2}{*}{0.069} \\
\hline High & $34.16(9.67)$ & \\
\hline
\end{tabular}

t-Test for independent groups.

*Low, score $\leq 6$ (percentile 10); high, score $=10$ (percentile 90).

†Low, score $\leq 3$ (percentile 10); high, score $=10$ (percentile 90).

łLow, score $\leq 35$ (percentile 10); high, score $=45$ (percentile 90).

$\mathrm{PI}$, pressure injury; PIPB, Pressure Injury Prevention Barriers. line with recent qualitative studies that have explored this phenomenon. ${ }^{49} 50$

Because the sample of nurses participating in this study was not obtained at random, it is possible that there was more participation from nurses who were more motivated towards PIs prevention, which may have led to an underestimation bias of the scores in the PIPB questionnaire.

Some limitations of the study have to be taken into account. This questionnaire was not tested for comprehension and relevance with the target population in the initial phase of development. These aspects should be explored in further research with nurses through quantitative or qualitative methods. The content validity was evaluated by the panel in the initial set of items, but not in the final 25-items version. However, since the items in the final version were included in the initial set, we think that this fact is unlikely to substantially alter the content validity of the questionnaire as a whole. Another limitation is that the temporal stability of the PIPB questionnaire was not evaluated through a test-retest procedure. Although the barriers to PIs prevention perceived in a service or unit are not expected to change in a short period of time, the stability of the scores obtained with the PIPB questionnaire should be explored in further studies.

Since the PIPB has been developed and assessed in hospitals, it is not known whether this questionnaire may be used in other settings, such as nursing homes, longterm care or community care; so additional studies are needed. Finally, it should be taken into account that the evaluation of the psychometric properties was done on the Spanish version of the PIPB questionnaire in a population of Spanish-speaking nurses. This is a restriction to the generalisation of the findings to different contexts and languages; the English version of the PIPB questionnaire should be further evaluated in different Englishspeaking populations before use.

\section{CONCLUSION}

The PIPB questionnaire is a short instrument to measure the nurses' perception of the barriers to PIs prevention. This questionnaire has initial evidence of content validity, internal consistency reliability and construct validity. However, further studies with a representative sample of the target population are needed to assess the relevance, comprehensiveness and comprehensibility of the questionnaire. Its four-factor structure allows calculation of an overall score, but also a score for each factor. The identification of perceived barriers is a major initial step in assessing whether those barriers are real barriers, and whether planning for improvements or changes is needed to enable PIs preventive measures to be applied.

Acknowledgements The authors thank all the professionals that participated in this study.

Contributors MDL-F and PLP-H conceived and designed the study. MDL-F, PLP-H, LP-A and IMC-S conducted statistical analysis and interpreted data for the work. MDL-F and PLP-H prepared the first draft of the manuscript. All authors critically 
reviewed the manuscript and approved the final version. MDL-F led the project under the supervision of PLP-H.

Funding The authors have not declared a specific grant for this research from any funding agency in the public, commercial or not-for-profit sectors.

Competing interests None declared.

Patient consent for publication Not required.

Ethics approval The protocol was approved by the Research Ethics Committee of Jaén, Spain (number: 2016/12-15).

Provenance and peer review Not commissioned; externally peer reviewed.

Data availability statement Data on questionnaire responses (anonymised) may be shared upon request to corresponding author.

Supplemental material This content has been supplied by the author(s). It has not been vetted by BMJ Publishing Group Limited (BMJ) and may not have been peer-reviewed. Any opinions or recommendations discussed are solely those of the author(s) and are not endorsed by BMJ. BMJ disclaims all liability and responsibility arising from any reliance placed on the content. Where the content includes any translated material, BMJ does not warrant the accuracy and reliability of the translations (including but not limited to local regulations, clinical guidelines, terminology, drug names and drug dosages), and is not responsible for any error and/or omissions arising from translation and adaptation or otherwise.

Open access This is an open access article distributed in accordance with the Creative Commons Attribution Non Commercial (CC BY-NC 4.0) license, which permits others to distribute, remix, adapt, build upon this work non-commercially, and license their derivative works on different terms, provided the original work is properly cited, appropriate credit is given, any changes made indicated, and the use is non-commercial. See: http://creativecommons.org/licenses/by-nc/4.0/.

ORCID iD

Pedro L Pancorbo-Hidalgo http://orcid.org/0000-0002-0092-6095

\section{REFERENCES}

1 Pressure Injury Stages. The National pressure injury Advisory panel (NPIAP), 2016. Available: https://npiap.com/page/ PressurelnjuryStages [Accessed 03 Apr 2020].

2 Moore Z, Avsar P, Conaty L, et al. The prevalence of pressure ulcers in Europe, what does the European data tell us: a systematic review. $J$ Wound Care 2019;28:710-9.

3 Pancorbo-Hidalgo P, García-Fernández F, Pérez-López C. Prevalence of pressure injuries and other dependence-related skin lesions in adult patients admitted to Spanish hospitals: the fifth national study in 2017. Gerokomos 2019;30:76-86.

4 Coyer F, Miles S, Gosley S, et al. Pressure injury prevalence in intensive care versus non-intensive care patients: a state-wide comparison. Aust Crit Care 2017;30:244-50.

5 Liu Y, Wu X, Ma Y, et al. The prevalence, incidence, and associated factors of pressure injuries among immobile inpatients: a multicentre, cross-sectional, exploratory descriptive study in China. Int Wound $J$ 2019;16:459-66.

6 Sari SP, Everink IH, Sari EA, et al. The prevalence of pressure ulcers in community-dwelling older adults: a study in an Indonesian City. Int Wound J 2019;16:534-41.

7 Bereded DT, Salih MH, Abebe AE. Prevalence and risk factors of pressure ulcer in hospitalized adult patients; a single center study from Ethiopia. BMC Res Notes 2018;11:847.

8 Bernardes RM, Caliri MHL. Pressure ulcer prevalence in emergency hospitals: a cross-sectional study. Online Braz J Nurs 2016;15:236-44.

9 Queiroz ACdeCM, Mota DDCdeF, Bachion MM, et al. [Pressure ulcers in palliative home care patients: prevalence and characteristics]. Rev Esc Enferm USP 2014;48:264-71.

10 Artico M, Dante A, D'Angelo D, et al. Prevalence, incidence and associated factors of pressure ulcers in home palliative care patients: a retrospective chart review. Palliat Med 2018;32:299-307.

11 Gorecki C, Brown JM, Nelson EA, et al. Impact of pressure ulcers on quality of life in older patients: a systematic review. J Am Geriatr Soc 2009;57:1175-83.

12 Padula WV, Delarmente BA. The National cost of hospital-acquired pressure injuries in the United States. Int Wound J 2019;16:634-40.

13 Ayello EA, Levine JM, Langemo D, et al. Reexamining the literature on terminal ulcers, scale, skin failure, and unavoidable pressure injuries. Adv Skin Wound Care 2019;32:109-21.
14 Never Events. Agency for healthcare research and quality. Available: https://psnet.ahrq.gov/primer/never-events [Accessed 03 Apr 2020].

15 Manderlier B, Van Damme N, Verhaeghe S, et al. Modifiable patientrelated factors associated with pressure ulcers on the sacrum and heels: secondary data analyses. J Adv Nurs 2019;75:2773-85.

16 Coleman S, Gorecki C, Nelson EA, et al. Patient risk factors for pressure ulcer development: systematic review. Int J Nurs Stud 2013;50:974-1003

17 Braden B, Bergstrom N. A conceptual schema for the study of the etiology of pressure sores. Rehabil Nurs 1987;12:8-16.

18 Coleman S, Nixon J, Keen J, et al. A new pressure ulcer conceptual framework. J Adv Nurs 2014;70:2222-34.

19 García-Fernández FP, Agreda JJS, Verdú J, et al. A new theoretical model for the development of pressure ulcers and other dependence-related lesions. J Nurs Scholarsh 2014;46:28-38.

20 Jaul E, Barron J, Rosenzweig JP, et al. An overview of co-morbidities and the development of pressure ulcers among older adults. BMC Geriatr 2018;18:305

21 European Pressure Ulcer Advisory Panel. National Pressure Injury Advisory Panel and Pan Pacific Pressure Injury Alliance. In: Haesler $\mathrm{E}$, ed. Prevention and treatment of pressure Ulcers/Injuries: quick reference guide. Perth, Australia: EPUAP/NPIAP/PPPIA, 2019.

22 Registered Nurses Association of Ontario. Assessment and management of pressure injuries for the interprofessional team. Third ed. Toronto, Ontario: Registered Nurses Association of Ontario, 2016

23 Avsar P, Patton D, O'Connor T, et al. Do we still need to assess nurses' attitudes towards pressure ulcer prevention? A systematic review. J Wound Care 2019;28:795-806.

24 Probst S, Holloway S, Rowan S, et al. Wound Curriculum for Nurses: Post-registration qualification wound management - european qualification framework level 6. J Wound Care 2019;28:S1-33.

25 Tschannen D, Anderson C. The pressure injury predictive model: a framework for hospital-acquired pressure injuries. J Clin Nurs 2020;29:1398-421.

26 Schindler CA. More than S.K.I.N. deep: decreasing pressure ulcer development in the pediatric intensive care unit. Marquette university, 2010. Available: https://epublications.marquette.edu/dissertations_ mu/85/ [Accessed 03 Apr 2020].

27 Moore Z, Price P. Nurses' attitudes, behaviours and perceived barriers towards pressure ulcer prevention. J Clin Nurs 2004;13:942-51.

28 Mwebaza I, Katende G, Groves S, et al. Nurses' knowledge practices, and barriers in care of patients with pressure ulcers in a Ugandan teaching hospital. Nurs Res Pract 2014;2014:973602

29 Källman U, Suserud B-O. Knowledge, attitudes and practice among nursing staff concerning pressure ulcer prevention and treatment - a survey in a Swedish healthcare setting. Scand J Caring Sci 2009;23:334-41.

30 López-Franco MD, Parra-Anguita L, Pancorbo-Hidalgo PL. Instruments for measuring attitudes and barriers to prevention: literature review. Gerokomos 2019;30:217-25.

31 Nursing and Innovation in healthcare. CuiDsalud. Universidad de Jaén (Spain). SECOACBA project. Available: https://cuidsalud.com/ en/inv/proyecto-secoacba/ [Accessed 14 May 2020].

32 Beitz JM. Overcoming barriers to quality wound care: a systems perspective. Ostomy Wound Manage 2001;47:56-64.

33 Reason J. Human error: models and management. BM 2000;320:768-70.

34 Merino Soto C. Intervalos de confianza asimétricos para El índice La validez de contenido: un programa visual basic para La V de Aiken. Anales de psicología 2009;25:169.

35 Polit DF, Beck CT. Developing and Testing Self-Report Scales. In: Polit DF, Beck CT, eds. Nursing research: generating and assessing evidence for nursing practice. 8 ed. China, Hong Kong: Lippincott Williams \& Wilkins, 2008: 474-505.

36 Beeckman D, Defloor T, Demarré L, et al. Pressure ulcers: development and psychometric evaluation of the attitude towards pressure ulcer prevention instrument (APuP). Int J Nurs Stud 2010;47:1432-41.

37 López-Franco MD, Parra-Anguita L, Comino-Sanz IM, et al. Attitudes of Spanish nurses towards pressure injury prevention and psychometric characteristics of the Spanish version of the APuP instrument. Int J Environ Res Public Health 2020;17:8543.

38 Muthén L, Muthén B. Mplus. The comprehensive modelling program for applied researchers: user's guide. Available: https://www. statmodel.com [Accessed 24 Aug 2020].

39 Lim CR, Harris K, Dawson J, et al. Floor and ceiling effects in the OHS: an analysis of the NHS PROMs data set. BMJ Open 2015;5:e007765.

40 Williams B, Onsman A, Brown T. Exploratory factor analysis: a fivestep guide for novices. Australas J Paramedicine 2010;8. 
41 Ledesma RD, Valero-Mora P. Determining the number of factors to retain in EFA: an easy-to-use computer program for carrying out parallel analysis. Pract Assess Res Evaluation 2007;12:1-11.

42 Watkins MW. Monte Carlo PCA for parallel analysis [computer software. Psych Associates ed. State College, PS, 2000.

$43 \mathrm{Hu} \mathrm{Li}$-tze, Bentler PM. Cutoff criteria for fit indexes in covariance structure analysis: conventional criteria versus new alternatives. Struct Equ Model 1999;6:1-55.

44 Streiner DL. Starting at the beginning: an introduction to coefficient alpha and internal consistency. J Pers Assess 2003;80:99-103.

45 Garza Hernández R, Meléndez Méndez MC, MÁ FH. Knowledge, attitude and barriers in nurses towards prevention measures of pressure ulcers. Rev Bras Nurse 2017;23:47-58. doi:10.1590/00347167-2016-0063
46 Drake J, Redfern WS, Sherburne E, et al. Pediatric skin care: what do nurses really know? J Spec Pediatr Nurs 2012;17:329-38.

47 Mirshekari L, Tirgari B, Forouzi MA. Intensive care unit nurses' perceived barriers towards pressure ulcer prevention in South East Iran. J Wound Care 2017; 26:145-51.

48 Berihu H, Wubayehu T, Teklu T, et al. Practice on pressure ulcer prevention among nurses in selected public hospitals, Tigray, Ethiopia. BMC Res Notes 2020;13:207.

49 Kuhnke JL, Keast D, Rosenthal S, et al. Health professionals perspectives on delivering patient-focused wound management: a qualitative study. J Wound Care 2019;28:S4-13.

50 Kaba E, Kelesi M, Stavropoulou A, et al. How Greek nurses perceive and overcome the barriers in implementing treatment for pressure ulcers: 'against the odds'. J Wound Care 2017;26:S20-6. 